\title{
Erratum to: Process Control
}

\section{Erratum to:}

J.-P. Corriou, Process Control, https://doi.org/10.1007/978-3-319-61143-3

The original versions of Chapters 12, 14 and 17 contained the following errors:

In Ch. 12, Figs. 12.5 and 12.6 were incorrect.

In Ch. 14, Figs. 14.12, 14.13 and 14.14 were incorrect.

In Ch. 17, the caption to Fig. 17.12 was in French.

These errors have been corrected.

The updated online version of this book can be found at https://doi.org/10.1007/978-3-31961143-3. 